\title{
Improving the Sensitivity of Deadlines with a Specific Asynchronous Scenario for Harmonic Periodic Tasks scheduled by FP
}

\author{
P. Meumeu Yomsi, Y. Sorel, D. de Rauglaudre \\ AOSTE Project-team \\ INRIA Paris-Rocquencourt \\ Le Chesnay, France \\ \{patrick.meumeu, yves.sorel, daniel.de_rauglaudre\}@inria.fr
}

\author{
L. George \\ LACSC \\ $E C E$ \\ Paris, France \\ lgeorge@ece.fr
}

\begin{abstract}
The aim of this paper is to address the problem of correctly dimensioning real-time embedded systems. It is well known that computers which control systems are greatly affected by delays and jitter occurring in the control loop. In the literature, a deadline reduction approach has been considered as one solution to reducing the jitter experienced by a task and therefore obtaining better loop stability in the control loop. Here, in order to improve the sensitivity of the deadlines, we propose another solution for reducing the deadline of a task when all tasks are scheduled with the Deadline Monotonic Algorithm. This is performed for a specific asynchronous scenario for harmonic periodic tasks. We compare the results to those for the synchronous scenario.
\end{abstract}

\section{Introduction}

In this paper, we consider the problem of correctly dimensioning real-time embedded systems. The correct dimensioning of a real-time system strongly depends on the determination of the tasks' Worst-Case Execution Times (WCETs). Based on the WCETs, Feasibility Conditions (FCs) ([1], [2], [3]) can be established to ensure that the timeliness constraints of all the tasks are always met when tasks are scheduled by a fixed or a dynamic priority driven scheduling algorithm. We consider an application composed of a periodic task set $\Gamma_{n}=\left\{\tau_{1}, \ldots, \tau_{n}\right\}$ of $n$ periodic tasks, scheduled with Fixed Priority (FP) preemptive scheduling. The classical definition of a periodic task $\tau_{i}$, is:

- $C_{i}$ : the Worst Case Execution Time (WCET) of $\tau_{i}$.

- $T_{i}$ : the period of $\tau_{i}$.

- $D_{i}$ : the relative deadline of $\tau_{i}$ (a task requested at time $t$ must be terminated by its absolute deadline $t+D_{i}$ ), where $D_{i} \leq T_{i}$.

A recent research area called sensitivity analysis aims at providing interesting information on the validity of feasibility conditions considering possible deviations of task WCETs
([4]), task periods ([4]), or task deadlines ([5]). This makes it possible, for example, to find a feasible task set, if the current one is not feasible, by modifying the task parameters or determining the impact of an architecture change on the feasibility of a task set. We are interested in the sensitivity of deadlines when tasks are scheduled with fixed priority Deadline Monotonic scheduling. Sensitivity analysis for deadlines has been considered for Earliest Deadline First (EDF) scheduling by ([5]) showing how to compute the minimum feasible deadlines such that deadline of any task $\tau_{i}$ is equal $\alpha D_{i}$, where $\alpha$ is reduction factor $0<\alpha \leq 1$. In ([6]), the space of feasible deadlines (D-space), a space of $n$ dimensions has been considered. Any task set having deadlines in the D-space is considered to be schedulable. To the knowledge of the authors, no work has been done on the sensitivity of deadlines for fixed priority scheduling.

Computer controlling systems are very much affected by delays and jitter occurring in the control loop. A deadline reduction has been considered by ([6]) as one solution to reducing the jitter experienced by a task and therefore obtaining better loop stability in the control loop. This paper proposes a solution reducing as much as possible the deadline of a task when tasks are scheduled with Deadline Monotonic.

With Deadline Monotonic, tasks are scheduled according to their relative deadlines. The smaller the relative deadline, the higher the priority. Starting from a schedulable task set, we want to characterize the minimum deadline reduction factor $0<\alpha \leq 1$ such that any task $\tau_{i}, i=1 \ldots n$ having a deadline $D_{i}=\alpha \times T_{i}$ is schedulable. $\alpha$ is such that any smaller reduction factor would lead to a non schedulable task set. We compare the value of $\alpha$ obtained in the worst case synchronous scenario (all the tasks are first released at the same time) to that obtained with a particular asynchronous scenario that we propose, and which has some interesting properties. We show that the minimum reduction factor obtained in our asynchronous scenario is always less than or equal to the minimum reduction factor obtained in the synchronous scenario. 
For Fixed Priority (FP) scheduling, necessary and sufficient FCs have been proposed in the case of non-concrete tasks where the first release times of the tasks can be arbitrary. A classical approach is based on the computation of the tasks' worst-case response times ([7], [2]). The worst-case response time, defined as the worst case time between the request time of a task and its latest completion time, is obtained in the worst case synchronous scenario corresponding to the case where all the tasks are released at the same time, and is computed by successive iterations. This worst case response time provides a bound on the response time valid for any other task first release times. A task set is declared feasible if for any task in the synchronous scenario, its worst case response time is less than or equal to its deadline. It can be shown that considering only non-concrete tasks can lead to a pessimistic dimensioning [8].

The complexity of this approach depends on the worst case response time computation complexity. In the case of deadlines less than or equal and to periods for all tasks, the worst-case response time $R_{i}$ of a task $\tau_{i}$ is obtained in the synchronous scenario for the first release of $\tau_{i}$ at time 0 and is the solution of the equation ([7]) $R_{i}=W_{i}\left(R_{i}\right)$, where $W_{i}(t)=C_{i}+\sum_{\tau_{j} \in h p(i)}\left[\frac{t}{T_{j}}\right\rceil C_{i}$ and $h p(i)$ denotes the set of tasks with a priority higher than or equal to that of $\tau_{i}$ except $\tau_{i}$ itself. The value of $R_{i}$ is computed by successive iterations and the number of iterations is bounded by $\left.1+\sum_{\tau_{j} \in h p(i)} \frac{D_{i}}{T_{j}}\right]$. The FC has been revisited by ([9]), which the authors showed that a necessary and sufficient feasibility condition for a task set is: $\exists t \in S$, such that $W_{i}(t) / t \leq 1$, where $S=\cup_{\tau_{j} \in h p(i)}\left\{k T_{j}, k \in N\right\} \cap\left[0, D_{i}\right]$. For any task $\tau_{i}$, the checking instants correspond to the arrival times of the tasks with a higher priority within the interval $\left[0, D_{i}\right]$. This feasibility has been improved by ([9]), where the authors showed how to reduce the time instants of $S$. For any task $\tau_{i}$, they show how to significantly reduce the number of checking instants during interval $\left[0, D_{i}\right]$ to at most $2^{i-1}$ times rather than $1+\sum_{\tau_{j} \in h p(i)}\left\lfloor\frac{D_{i}}{T_{j}}\right\rfloor$. When deadlines and periods are independent, ([2]) shows that the worstcase response times of a sporadic task $\tau_{i}$ is not necessarily obtained for the first activation request of $\tau_{i}$ at time 0 . The number of activations to consider is $1+\left\lfloor\frac{L_{i}}{T_{i}}\right\rfloor$, where $L_{i}$ is the length of the worst-case level- $\tau_{i}$ busy period defined in ([10]) as the longest period of processor activity running tasks of priority higher than or equal to $\tau_{i}$ in the synchronous scenario. It can be shown that $L_{i}=\sum_{\tau_{j} \in h p(i) \cup \tau_{i}}\left\lceil\frac{L_{i}}{T_{j}}\right\rceil C_{j}$. From its definition, $L_{i}$ is bounded by:

$$
\operatorname{Min}\left\{\begin{array}{c}
\sum_{\tau_{j} \in h p(i) \cup \tau_{i}} \frac{C_{j}}{1-\sum_{\tau_{j} \in h p(i) \cup \tau_{i}} \frac{C_{j}}{T_{j}}}, \\
\sum_{\tau_{j} \in h p(i) \cup \tau_{i}} \frac{C_{j}}{T_{j}} \cdot P
\end{array}\right\}([3]) .
$$

where $P=\operatorname{LCM}\left(T_{1}, \ldots, T_{n}\right)$ and it leads to a pseudopolynomial time complexity for the feasibility conditions.

This is an interesting approach as it provides a pseudopolynomial time complexity but it may lead to a pessimistic dimensioning as the synchronous scenario might not be likely to occur.

In order to improve the schedulability of the systems, offset strategies on the first release times of the tasks have been considered. A system where offsets are imposed is called an asynchronous systems. ([8]) shows significant feasibility improvements considering offsets. Simulations show that the number of feasible schedulable systems with offsets (while unfeasible in the synchronous case) increases with the number of tasks for a processor load of 0.8 and ranges from $40.5 \%$ to $97 \%$ for different offset assignment strategies. This percentage strongly decreases when the load is high (tends to 1 ).

With asynchronous tasks, ([11]) shows that for a given offset assignment, the schedulability of the tasks must be checked in the interval $\left[0, \max _{i=1 \ldots n}\left(O_{i}\right)+2 P\right)$ where $P$ is the least common multiple of the tasks and $O_{i}$ is the offset of task $\tau_{i}$, leading to an exponential time complexity. To provide less pessimistic FCs, it is furthermore mandatory to prove that the offsets will not result later in a synchronous scenario. This problem is referred to as the $\mathrm{K}$-simultaneous congruence problem in the state of the art ([11]). This feasibility result has been significantly improved by ([12]) showing that the interval to check the feasibility of a periodic task set with offsets can be reduced to $\left[0, \max _{i=1 \ldots n}\left(O_{i}\right)+P\right)$.

Furthermore, ([11]) proves the non optimality of Deadline Monotonic scheduling for asynchronous systems when tasks deadlines are less than or equal to periods. An optimal priority assignment can be obtained in $O\left(n^{2}\right)$ using the Audsley procedure ([13]).

A particular case denoted offset free systems corresponds to the case where offsets can be chosen arbitrarily. An optimal offset assignment is given in ([14]). An offset assignment is optimal if it can find a schedulable offset whenever a feasible assignment exists. The complexity of the offset assignment algorithm is exponential and is in $O\left(\left(\max _{2 \leq j \leq n} T_{j}\right)^{n-1}\right)$. The offset of task $\tau_{1}$ is set to 0 . Different offset strategies heuristics have been considered in the literature. Among them, we can cite the dissimilar offset assignment proposed by ([14]) that consists in shifting (computing a distance between the offsets) the offset of the tasks to be as far as possible from the synchronous scenario. The algorithm sorts the couple of tasks $\left(\tau_{i}, \tau_{j}\right)$ by decreasing values of $\operatorname{gcd}\left\{T_{i}, T_{j}\right\}$ such that the distance belongs to $\left[0, \operatorname{gcd}\left\{T_{i}, T_{j}\right\}\right)$. The dissimilar offset assignment significantly reduces the number of offsets to consider, leading to a complexity in $O\left(n^{2} .\left(\log \left(\max _{i \in[1, n]} T_{i}\right)+\log \left(n^{2}\right)\right)\right)$. Other offset assignment strategies have been considered by ([8]) using the Audsley procedure to determine the subset of 
tasks of $\tau$ that can be feasibly scheduled in the synchronous scenario (setting their offset to 0 ). The offsets are only computed for the subset of tasks that are unfeasible with the Audsley procedure in the synchronous case. The authors consider different criteria to assign the offsets, based on the criteria used to sort the couple of tasks $\left(\tau_{i}, \tau_{j}\right)$. The complexity is the same as that of the dissimilar offset assignment.

In this paper we consider a particular asynchronous harmonic concrete task set where $\forall 2 \leq i \leq n, T_{i-1} \mid T_{i}$ (i.e. there exists $k \in Z$ such that $T_{i}=k T_{i-1}$ ) with particular offsets. In the case of non-concrete harmonic tasks, when tasks are scheduled with Rate Monotonic (the shorter the period, the higher the priority) and in the case deadlines are equal to periods, a necessary and sufficient condition for the feasibility of such a system is given by $U=\sum_{i=1 \ldots n} \frac{C_{i}}{T_{i}} \leq 1$ (see [15]). This potentially proves the benefits of considering harmonic tasks getting better feasibility conditions. This property does not hold when deadlines can be shorter than periods. In this case we show how to determine in $\mathcal{O}(n)$ the offset of the tasks to obtain a pseudo-polynomial time feasibility condition instead of an exponential one. In the case of asynchronous tasks, the worst case response time cannot be computed with a recursive equation as for the synchronous tasks. This is due to the fact that with offsets, there is not necessarily a continuous busy period from time 0 to the release time of a task. In this paper we investigate a new approach to compute the worst case response time of a task based on the Mesoid approach. This approach was first introduced by ([16]) in the context of real-time scheduling with preemption cost. This approach does not require a continuous busy period to compute the worst case response times of the tasks. We propose a particular offset assignment, such that the worst case response time of any task is obtained for its second request time, providing an exponential time improvement in the complexity of the FCs.

The rest of the paper is organized as follows. In section 2 , we introduce the concepts and notations and establish important properties for the particular asynchronous scen ario that we consider. We consider harmonic periods. We show that, using this particular scenario, the worst case response time of every task is obtained for its second instance. In section 3, we introduce the concept of Mesoid which is used to compute the worst case response time of an asynchronous task set. In section 4, we give an algorithm for the computation of the worst case response time of any task in our asynchronous scenario, then we show how to compute the minimum deadline reduction factor. An example is given in order to compare the deadline reduction factor obtained with our asynchronous scenario to that in the synchronous scenario. We provide experimental results in section 5 based on extensive simulations comparing the deadline reduction factor for several load configurations in both the synchronous and in our asynchronous scenario. Finally we conclude the paper in section 6 .

\section{Properties of the asynchronous harmonic task set}

\subsection{Concepts and notations}

We recall classical results in the uniprocessor context for real-time scheduling.

- Time is assumed to be discrete (task arrivals occur and task executions begin and terminate at clock ticks; the parameters used are expressed as multiples of the clock tick); in [17], it is shown that there is no loss of generality with respect to feasibility results by restricting the schedules to be discrete, once the task parameters are assumed to be integers (multiples of the clock tick) i.e. a discrete schedule exists, if and only if a continuous schedule exists.

- A task set is said to be valid with a given scheduling policy if and only if no task occurrence ever misses its absolute deadline with this scheduling policy.

- $U=\sum_{i=1}^{n} \frac{C_{i}}{T_{i}}$ is the processor utilization factor associated to the task set $\Gamma_{n}$, i.e., the fraction of processor time spent in the execution of the task set ([18]). If $U>1$, then no scheduling algorithm can meet the tasks' deadlines.

- The synchronous scenario corresponds to the scenario where all the tasks are released at the same time (at time 0).

The model depicted in figure 1 is Liu \& Layland's pioneering model [18] for systems executed on a single processor.

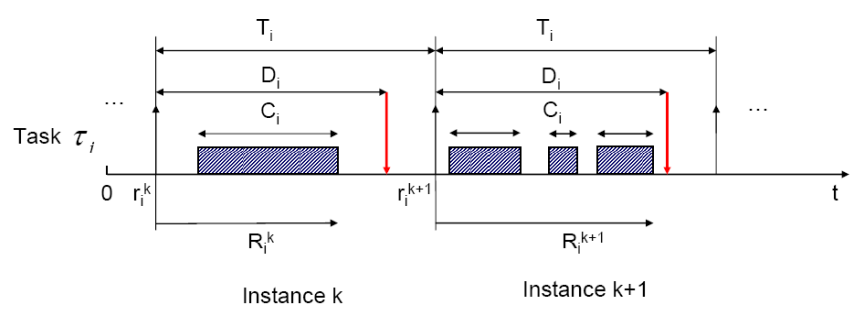

Figure 1. Model

Throughout the paper, we assume that all timing characteristics are non-negative integers, i.e. they are multiples of some elementary time interval (for example the "CPU tick", the smallest indivisible CPU time unit):

We introduce several notations for a periodic task $\tau_{i}=$ $\left(C_{i}, D_{i}, T_{i}\right)$ used to compute the worst case response time of a task:

- $\tau_{i}^{k}$ : The $k^{t h}$ instance of $\tau_{i}$ 
- $r_{i}^{1}$ : Release time of the first instance of $\tau_{i}$

- $r_{i}^{k}=r_{i}^{1}+(k-1) T_{i}$ : Release time of $\tau_{i}^{k}$

- $R_{i}^{k}$ : Response time of $\tau_{i}^{k}$ released at time $r_{i}^{k}$

- $R_{i}$ : Worst-case response time of $\tau_{i}$

\subsection{The specific asynchronous scenario}

Now, we give some interesting properties which are satisfied by the specific asynchronous scenario we propose which lead to the conclusion that the worst case response time of a task in our asynchronous scenario is obtained for any task for its second release.

In this section we assume that the relative deadline for each task equals its period, i.e. $D_{i}=T_{i}$. This assumption will be weakened in section 4 .

We first show in lemma 1 that with harmonic asynchronous tasks, two instances belonging to any two tasks can never be released at the same time if their release times are not equal modulo their periods.

Lemma 1: Let $\Gamma_{n}=\left\{\tau_{1}, \tau_{2}, \cdots, \tau_{n}\right\}$ be a system of $n$ independent harmonic (i.e. $T_{i} \mid T_{i+1}, \forall i \in\{1, \cdots, n-1\}$ ) preemptive tasks ordered by decreasing priorities $\left(T_{i} \leq\right.$ $\left.T_{i+1}, \forall i \in\{1, \cdots, n-1\}\right)$.

If there exist two tasks $\tau_{i}, \tau_{j} \in \Gamma_{n},(i<j)$ such that $r_{j}^{1} \neq r_{i}^{1} \bmod \left[T_{i}\right]^{1}$, then $\nexists k, l \geq 0$ such that $r_{j}^{k}=r_{i}^{l}$.

Proof: (by contradiction) Let us assume that there exist two tasks $\tau_{i}, \tau_{j} \in \Gamma_{n},(i<j)$ such that $r_{j}^{1} \neq r_{i}^{1} \bmod \left[T_{i}\right]$, and $\exists k, l \geq 0$ such that $r_{j}^{k}=r_{i}^{l}$.

$$
\begin{aligned}
r_{j}^{k}=r_{i}^{l} & \Leftarrow r_{j}^{1}+(k-1) T_{j}=r_{i}^{1}+(l-1) T_{i} \\
& \Leftarrow r_{j}^{1}=r_{i}^{1}+(l-1) T_{i}-(k-1) T_{j} \\
& \Leftarrow r_{j}^{1}=r_{i}^{1} \bmod \left[T_{i}\right] \text { as } T_{i} \mid T_{j} .
\end{aligned}
$$

Contradicts the hypothesis and thus, ends the proof.

We now show in theorem 1 that from the point of view of any task in the system, the schedule repeats identically from the second instance.

(inspired by theorem 2.48 in [19])

Let $\Gamma_{n}=\left\{\tau_{1}, \tau_{2}, \cdots, \tau_{n}\right\}$ be a system of $n$ asynchronous independent periodic preemptive tasks ordered by decreasing priorities $\left(T_{i} \leq T_{i+1}, \forall i \in\{1, \cdots, n-1\}\right)$. Let $r_{1}^{1}, r_{2}^{1}, \cdots, r_{n}^{1}$ be respectively the release time of their first instances. Let $\left(s_{i}\right)_{1 \leq i \leq n}$ be the sequence inductively defined by

$$
\left\{\begin{array}{l}
s_{1}=r_{1}^{1} \\
s_{i}=r_{i}^{1}+\left\lceil\frac{\left(s_{i-1}-r_{i}^{1}\right)^{+}}{T_{i}}\right\rceil \cdot T_{i} \quad \forall i \in\{2, \cdots, n\}
\end{array}\right.
$$

Then,

if $\Gamma_{n}$ is schedulable up to $s_{n}+H_{n}$, with $H_{n}=$ $\operatorname{LCM}\left(T_{1}, T_{2}, \cdots, T_{n}\right)$ and $x^{+}=\max \{x, 0\}$, then $\Gamma_{n}$ is schedulable and periodic from $s_{n}$ with period $H_{n}$.

1. Given $a, b, c \in Z: a=b \bmod [c]$ means that there exists $d \in Z$ such that $a=b+c \dot{d}$.
Proof: (By induction on the number of tasks $n$ ) The property is straightforward for the simple case where $n=1$ : indeed, the schedule for task $\tau_{1}$ is periodic of period $T_{1}$ from its first release $\left(s_{1}=r_{1}^{1}\right)$ since $C_{1} \leq T_{1}$, otherwise the deadline of the first instance is missed. Let us now assume that the property is true up to $n=i-1$ and $\Gamma_{i}=\left\{\tau_{1}, \tau_{2}, \cdots, \tau_{i}\right\}$ is schedulable up to $s_{i}+H_{i}$, with $H_{i}=\operatorname{LCM}\left(T_{1}, T_{2}, \cdots, T_{i}\right)$. Notice that $s_{i}$ is the first release time of task $\tau_{i}$ after (or at) $s_{i-1}$. We have $s_{i}+H_{i} \geq s_{i-1}+H_{i-1}$ and by induction hypothesis, the subset $\Gamma_{i-1}=\left\{\tau_{1}, \tau_{2}, \cdots, \tau_{i-1}\right\}$ is schedulable and periodic from $s_{i-1}$ of period $H_{i-1}$. As tasks are ordered by priority, the instances of the first ones are not changed by the requests of task $\tau_{i}$ and the schedule repeats at time $s_{i}+\operatorname{lcm}\left(H_{i-1}, T_{i}\right)=s_{i}+H_{i}$. Consequently, $\Gamma_{i}=$ $\left\{\tau_{1}, \tau_{2}, \cdots, \tau_{i}\right\}$ is schedulable and its schedule repeats from $s_{i}$ with period $H_{i}$.

We now characterize the asynchronous scenario we consider in this paper in corollary 1 . This leads to providing a simple method for computing the worst response time of each task in section 3 by using corollary 2 , and then a pseudo polynomial FC detailed in section 4.1.

Corollary 1: From the point of view of any task $\tau_{i}$ of a schedulable system $\Gamma_{n}=\left\{\tau_{1}, \tau_{2}, \cdots, \tau_{n}\right\}$ ordered by decreasing priorities $\left(T_{i} \leq T_{i+1}, \forall i \in\{1, \cdots, n-1\}\right)$ such that $T_{i} \mid T_{i+1}$ and $r_{i+1}^{1}=r_{i}^{1}-C_{i+1}$, the schedule is periodic from the second instance with period $H_{i}=T_{i}$.

Proof: (By induction on the index $i$ of the task) Let us consider a task $\tau_{i}$ of a schedulable system $\Gamma_{n}=$ $\left\{\tau_{1}, \tau_{2}, \cdots, \tau_{n}\right\}$, we assume that $T_{i} \mid T_{i+1}$ and $r_{i+1}^{1}=$ $r_{i}^{1}-C_{i+1}, \quad \forall i \geq 1$. Thanks to the previous theorem, it is sufficient to prove that $s_{i}-r_{i}^{1}=T_{i}, \quad \forall i \geq 2$. This is done by induction on $i$.

The property is straightforward for the simple case where $i=2$ : indeed, as $C_{2} \leq T_{2}$ and $H_{2}=\operatorname{LCM}\left(T_{1}, T_{2}\right)=T_{2}$, the schedule for task $\tau_{2}$ is periodic of period $T_{2}$ from its second release since $s_{2}=r_{2}^{1}+\left\lceil\frac{\left(s_{1}-r_{2}^{1}\right)^{+}}{T_{2}}\right\rceil \cdot T_{2}=r_{2}^{1}+$ $\left\lceil\frac{C_{2}}{T_{2}}\right\rceil \cdot T_{2}=r_{2}^{1}+T_{2}$ is the first release time of task $\tau_{2}$ after (or at) $s_{1}=r_{1}^{1}$. Let us now assume that the property is true up to index $i-1$ and $\Gamma_{i}=\left\{\tau_{1}, \tau_{2}, \cdots, \tau_{i}\right\}$ is schedulable. Thanks to the previous theorem, we have

$s_{i}=r_{i}^{1}+\left\lceil\frac{\left(s_{i-1}-r_{i}^{1}\right)^{+}}{T_{i}}\right\rceil \cdot T_{i}=r_{i}^{1}+\left\lceil\frac{\left(T_{i-1}+r_{i-1}^{1}-r_{i}^{1}\right)^{+}}{T_{i}}\right\rceil \cdot T_{i}$

by induction hypothesis.

Thus, $s_{i}=r_{i}^{1}+\left\lceil\frac{\left(T_{i-1}+C_{i}\right)^{+}}{T_{i}}\right\rceil \cdot T_{i}$ since $r_{i-1}^{1}=r_{i}^{1}+C_{i}$. Now, as $0<T_{i-1}+C_{i}<T_{i}$ due to the scenario imposed to the first instance of each task and the fact that $T_{i-1} \mid T_{i}$, then we obtain $s_{i}=r_{i}^{1}+T_{i}$.

Corollary 2: The worst response time $R_{i}$ of each task $\tau_{i}$ is obtained in the second instance and is equal to that in all 
instances greater than 2 .

Proof: Immediately follows from corollary 1 and the fact that $R_{i}^{1}=C_{i}$ by construction, $R_{i}^{k} \geq C_{i} \quad \forall k \geq 1$, and we consider harmonic tasks.

We now suppose in the following the asynchronous task set defined in corollary 1 . We now present the Mesoid approach used to compute the worst case response time of periodic tasks in an asynchronous task set.

\section{Worst case response time: the Mesoid ap- proach}

In this section, we provide the method for computing the worst response time of each task in order to check its schedulability. Actually, three classical methods may be used to do so: the utilisation factor of the processor ([20]), the worst response time of each task, or the processor demand ([21]). In this paper, we have chosen to use the second approach as it provides a schedulability condition for each task individually. The main idea behind the Mesoid approach is filling some available time units left by the schedule of higher priority tasks with executed time units corresponding to the execution time of the current task. Since the worst response time is obtained in the second instance w.r.t. corollary 2, we will achieve this goal by applying the method described in [16] to a system where all tasks are not released simultaneously and where the cost of a preemption is assumed to be zero. This method, contrary to those proposed in ([22], [3], [23]), is of lesser complexity since it is not necessary to determine the releases of every task w.r.t. those of higher priority tasks.

As we are in a fixed priority context the proposed method checks for the schedulability of each task by computing its worst response time, from the task with the highest priority to that with the lowest priority. Hence, from the point of view of any task $\tau_{i}$ of a system $\Gamma_{n}=\left\{\tau_{1}, \tau_{2}, \cdots, \tau_{n}\right\}$ ordered by decreasing priorities $\left(T_{i-1} \leq T_{i}, \forall i \in\{2, \cdots, n\}\right)$ such that $T_{i-1} \mid T_{i}$ and $r_{i}^{1}=r_{i-1}^{1}-C_{i}$, the elapsed duration between the release of the second instance and the first release $r_{i-1}^{1}$ of task $\tau_{i-1}$ is given by $T_{i}-C_{i}$. Before providing the computation method of the worst case response time, we provide some necessary definitions below.

\subsection{Definitions}

All the definitions and terminologies used in this section are directly inspired by ([16]) and are applied here to the case of a model where the cost of preemption is assumed to be zero. From the point of view of any task $\tau_{i}$, the hyperperiod at level $i, H_{i}$, is given by $H_{i}=L C M\left\{T_{j}\right\}_{\tau_{j} \in s p\left(\tau_{i}\right)}=$ $T_{i}$ as $T_{i-1} \mid T_{i}$ for every $i \in\{2, \cdots, n\}$, and $\operatorname{sp}\left(\tau_{i}\right)$ is the set of tasks with a period shorter than that of task $\tau_{i}$. Without any loss of generality we assume that the first task $\tau_{1}$ starts its execution at time $t=0$ and all tasks have different periods. Since at each level the schedule repeats indefinitely from the second instance thanks to corollary 1 , it is sufficient to perform the scheduling analysis in the interval $\left[r_{i}^{1}+T_{i}, r_{i}^{1}+2 T_{i}\right]$ for task $\tau_{i}$ as its response time in its first instance equals its WCET.

We proceed the schedule from the task with the shortest period towards the task with the longest period. Thus, at each level in the scheduling process the goal is to fill available time units in the previous schedule, obtained up to now, with slices of the WCET of the current task, hence we obtain the next current schedule. Consequently, we represent the previous schedule of every instance $\tau_{i}^{k}$ of the current task $\tau_{i}=\left(C_{i}, T_{i}\right)$ by an ordered set of $T_{i}$ time units where some have already been executed because of the execution of tasks with shorter periods, and the others are still available for the execution of task $\tau_{i}$ in that instance. We call this ordered set which describes the state of each instance $\tau_{i}^{k}$ the $\mathcal{M}_{i}^{k}$ $T_{i}$-mesoid. More details on the definition of a $T_{i}$-mesoid are given in [16]. For the current task $\tau_{i}=\left(C_{i}, T_{i}\right)$, there are as many $T_{i}$-mesoids as instances. We call $\mathcal{M}_{i}^{b, 2}$ the $T_{i}$-mesoid corresponding to the second instance of task $\tau_{i}$ before being scheduled in the current schedule. The process used to build $\mathcal{M}_{i}^{b, 2}$ for task $\tau_{i}$ will be detailed later in this subsection. Still, from the point of view of task $\tau_{i}$, we define for the mesoid $\mathcal{M}_{i}^{b, 2}$ the corresponding universe $X_{i}^{2}$ to be the ordered set, compatible with that of the mesoid, which consists of all the availabilities of $\mathcal{M}_{i}^{b, 2}$. That is to say, all the possible values that $C_{i}$ can take in $\mathcal{M}_{i}^{b, 2}$. Task $\tau_{i}$ will be said to be potentially schedulable if and only if

$$
C_{i} \in X_{i}^{2} \quad \forall i \in\{1, \cdots, n\}
$$

This equation verifies that $C_{i}$ belongs to the universe at level $i$. If it does not, then the system is clearly not schedulable. When equation (2) holds for a given task $\tau_{i}$, we call $\mathcal{M}_{i}^{a, 2}$ the $T_{i}$-mesoids corresponding to the second instance of task $\tau_{i}$ after $\tau_{i}$ has been scheduled. $\mathcal{M}_{i}^{a, 2}$ is a function of $\mathcal{M}_{i}^{b, 2}$ which itself is a function of $\mathcal{M}_{i-1}^{a, 2}$, both detailed as follows.

Let $f$ be the function such that $\mathcal{M}_{i}^{b, 2}=f\left(\mathcal{M}_{i-1}^{a, 2}\right)$ which transforms the $T_{i-1}$-mesoid after task $\tau_{i-1}$ has been scheduled at level $i-1$ into the $T_{i}$-mesoid before task $\tau_{i}$ is scheduled at level $i$.

As mentioned in [16], a mesoid consists only of time units already executed denoted by " $e$ " and time units still available denoted by " $a$ ". Moreover, the cardinal of a mesoid is equal to the period of the task under consideration whatever the level is. As such, the function $f$ transforms a time unit already executed (resp. still available) in $\mathcal{M}_{i-1}^{a, 2}$ into a time unit already executed (resp. still available) in $\mathcal{M}_{i}^{b, 2}$ by following an index $\psi$ which enumerates, according to naturals, the time units (already executed or still available) in $\mathcal{M}_{i-1}^{a, 2}$ of task $\tau_{i-1}$ after $\tau_{i-1}$ has been scheduled. As the elapsed duration between the release of the second instance of task $\tau_{i}$ and the release of the first instance of $\tau_{i-1}$ is $T_{i}-C_{i}$, then $\psi$ 
starts from the time unit right after $\gamma_{i}=T_{i}-C_{i} \bmod \left[T_{i-1}\right]$ time units in the mesoid $\mathcal{M}_{i-1}^{a, 2}$ towards the last time unit, and then circles around to the beginning of the mesoid $\mathcal{M}_{i-1}^{a, 2}$ again, until we get the $T_{i}$-mesoid $\mathcal{M}_{i}^{b, 2}$. This $T_{i^{-}}$ mesoid is obtained when $\psi=T_{i}$. Indeed, the previous schedule at level $i$ (the schedule obtained at level $i-1$ ) consists of $H_{i-1}=T_{i-1}$ time units whereas the schedule of the current task $\tau_{i}$ is computed upon $H_{i}=T_{i}$ time units. Thus, that amounts to extending the previous schedule from $T_{i-1}$ to $T_{i}$ time units by identically repeating the previous schedule as often as necessary to obtain $H_{i}$ time units. Due to the particular releases of the first instance of each task, i.e. $r_{i+1}^{1}=r_{i}^{1}-C_{i+1} \forall i \in\{1, \cdots, n-1\}$, notice that index $\psi$ in contrast to index $\zeta$ used in [16] which started from the first time unit, starts from the time unit right after $\gamma_{i}=T_{i}-C_{i} \bmod \left[T_{i-1}\right]$ time units in the mesoid $\mathcal{M}_{i-1}^{a, 2}$. Since $\tau_{1}$ is the task with the shortest period, then $\operatorname{sp}\left(\tau_{1}\right)=\left\{\tau_{1}\right\}$. Because $\tau_{1}$ is never preempted, we have $\mathcal{M}_{1}^{b, 2}=\left\{1,2, \cdots, T_{1}\right\}$ and therefore we obtain $\mathcal{M}_{1}^{a, 2}=\left\{\left(C_{1}\right), 1,2, \cdots, T_{1}-C_{1}\right\}$.

Let $g$ be the function such that $\mathcal{M}_{i}^{a, 2}=g\left(\mathcal{M}_{i}^{b, 2}\right)$ which transforms the $T_{i}$-mesoid $\mathcal{M}_{i}^{b, 2}$ before task $\tau_{i}$ has been scheduled at level $i$ into the $T_{i}$-mesoid $\mathcal{M}_{i}^{a, 2}$ after task $\tau_{i}$ has been scheduled at level $i$.

\subsection{Worst case response time with a Mesoid}

For the $T_{i}$-mesoid $\mathcal{M}_{i}^{b, 2}$, we will compute the response time $R_{i}^{2}$ of task $\tau_{i}$ in the second instance by adding to the WCET $C_{i}$ all the consumptions appearing in that $T_{i}$-mesoid before the availability corresponding to $C_{i}$ [16]. This yields the worst-case response time $R_{i}$ of task $\tau_{i}$ since at each level the schedule becomes periodic from the second instance, that is to say $R_{i}^{k}=R_{i}^{2} \forall k \geq 2$, and $R_{i}^{1}=C_{i} \forall i \geq 1$.

Now we can build $\mathcal{M}_{i}^{a, 2}=g\left(\mathcal{M}_{i}^{b, 2}\right)$ : function $g$ transforms a time unit already executed in $\mathcal{M}_{i}^{b, 2}$ into a time unit already executed in $\mathcal{M}_{i}^{a, 2}$, and transforms a time unit still available into either a time unit still available or a time unit already executed w.r.t. the following condition. We use an index which enumerates according to numerals the time units in $\mathcal{M}_{i}^{b, 2}$ from the first to the last one, at each step in the incremental process, if the current value of the index is less than or equal to $R_{i}^{2}$, function $g$ transforms the time unit still available into a time unit already executed due to the execution of instance $\tau_{i}^{2}$, otherwise $g$ transforms it into a time unit still available. Indeed, function $g$ fills available time units in the current schedule with slices of the WCET in each $T_{i}$-mesoid, leading to the previous schedule for the next task at level $i+1$ w.r.t. priorities. To summarize, for every task $\tau_{i}$, we have

$\tau_{i}:\left\{\begin{array}{l}\mathcal{M}_{i}^{b, 2}: T_{i} \text {-mesoid before } \tau_{i} \text { is scheduled at level } i \\ \mathcal{M}_{i}^{a, 2}: T_{i} \text {-mesoid after } \tau_{i} \text { is scheduled at level } i .\end{array}\right.$

\section{Deadline reduction factor}

\subsection{Worst case response time computation}

The approach proposed here leads to a new schedulability condition for harmonic hard real-time systems. This condition is new in the sense that besides providing a necessary and sufficient schedulability condition, it also reduces the feasibility interval for a given harmonic asynchronous system.

In the scheduling process, at each level $i$, the basic idea consists in filling availabilities in the mesoid $\mathcal{M}_{i}^{b, 2}$ before task $\tau_{i}$ is scheduled, with slices of its WCET. This is why it is fundamental to calculate the corresponding response time. This yields the worst case response time and allows us to conclude on the schedulability of task $\tau_{i}$ w.r.t. priorities. In the case where $\tau_{i}$ is schedulable, we build $\mathcal{M}_{i}^{a, 2}$, after $\tau_{i}$ has been scheduled, in order to check the schedulability of the next task, and so on, otherwise the system is not schedulable. Thanks to everything we have presented up to now, $\tau_{1}$ is scheduled first and $r_{1}^{1}=0$. The latter statement implies that before $\tau_{1}$ is scheduled, its WCET can potentially take any value from 1 up to the value of its period $T_{1}$. Since task $\tau_{1}$ is never preempted, then $\mathcal{M}_{1}^{b, 2}=\left\{1,2, \cdots, T_{1}\right\}$ and $X_{1}^{2}=\left\{1,2, \cdots, T_{1}\right\}$. In addition, its response time is also equal to $C_{1}$. Consequently, the corresponding $T_{1}$-mesoids associated to task $\tau_{1}$ are given by

$$
\tau_{1}:\left\{\begin{array}{l}
\mathcal{M}_{1}^{b, 2}=\left\{1,2, \cdots, T_{1}\right\} \\
\mathcal{M}_{1}^{a, 2}=\left\{\left(C_{1}\right), 1,2, \cdots, T_{1}-C_{1}\right\}
\end{array}\right.
$$

We assume that the first $i-1$ tasks with $2 \leq i \leq n$ have already been scheduled, i.e. the $T_{i-1}$-mesoid $\overline{\mathcal{M}}_{i-1}^{a, \overline{2}}$ of task $\tau_{i-1}$ is known, and that we are about to schedule task $\tau_{i}$.

As explained in the previous section, the $T_{i}$-mesoid $\mathcal{M}_{i}^{b, 2}=f\left(\mathcal{M}_{i-1}^{a, 2}\right)$ of task $\tau_{i}$ is built thanks to index $\psi$ on $\mathcal{M}_{i-1}^{a, 2}$ of task $\tau_{i-1}$ without forgetting to start from the time unit right after $\gamma_{i}=T_{i}-C_{i} \bmod \left[T_{i-1}\right]$ time units rather than the first time unit as in [16]. Again this is due to the particular release of the first instances of tasks: $r_{i}^{1}=r_{i-1}^{1}-C_{i}$. We can therefore determine the universe $X_{i}^{2}$ when the $T_{i-1}$-mesoid $\mathcal{M}_{i-1}^{a, 2}$ is known. Unless the system is not schedulable, i.e. $C_{i} \notin X_{i}^{2}$, we assume that task $\tau_{i}$ is potentially schedulable, i.e. $C_{i} \in X_{i}^{2}$. The response time $R_{i}^{2}$ of task $\tau_{i}$ in its $k^{t h}$ instance (with $k \geq 2$ ), i.e. in the $k^{t h} T_{i}$-mesoid will be obtained by summing $C_{i}$ with all consumptions prior to $C_{i}$ in the corresponding mesoid. The worst-case response time $R_{i}$ of task $\tau_{i}$ will then be given by

$$
R_{i}=R_{i}^{2}
$$

This equation leads us to say that task $\tau_{i}$ is schedulable if and only if

$$
R_{i} \leq T_{i}
$$


If for task $\tau_{i}$ expression (3) holds, then $\mathcal{M}_{i}^{a, 2}=g\left(\mathcal{M}_{i}^{b, 2}\right)$ will be deduced as explained in the previous section. For the sake of clarity, whenever there are two consecutive consumptions in a mesoid, this amounts to considering only one consumption which is the sum of the previous consumptions. That is to say that after determining the response time of task $\tau_{i}$ in its $k^{t h}$ mesoid, if $\mathcal{M}_{i}^{a, k}=\left\{\left(c_{1}\right),\left(c_{2}\right), 1,2, \cdots\right\}$, then this is equivalent to $\mathcal{M}_{i}^{a, k}=\left\{\left(c_{1}+c_{2}\right), 1,2, \cdots\right\}$.

Below, we present our scheduling algorithm which, for a given task, on the one hand first determines the value of $\gamma_{i}=T_{i}-C_{i} \bmod \left[T_{i-1}\right]$ relative to priorities, then, on the other hand the schedulability condition. Recall that the elapsed duration between the release of the second instance and the first release is $T_{i}-C_{i}$. The scheduling algorithm has the following nine steps. Since the task with the shortest period, namely task $\tau_{1}$, is never preempted, the loop starts from the index of the task with the second shortest period, namely task $\tau_{2}$ as the schedule proceeds towards tasks with longer periods.

1: for $i=2$ to $n$ do

2: Determine the release time of the first instance of task $\tau_{i}$ :

$$
r_{i}^{1}=r_{i-1}^{1}-C_{i}
$$

and compute $\gamma_{i}=T_{i}-C_{i} \bmod \left[T_{i-1}\right]$ of the second instance of $\tau_{i}$ w.r.t. $\tau_{i-1}$.

3: $\quad$ Build the $T_{i}$-mesoid $\mathcal{M}_{i}^{b, 2}=f\left(\mathcal{M}_{i-1}^{a, 2}\right)$ of task $\tau_{i}$ before it is scheduled. This construction is based on a modulo $T_{i}$ arithmetic using index $\psi$ on $\mathcal{M}_{i-1}^{a, 2}$ without forgetting to start from the time unit right after $\gamma_{i}=$ $T_{i}-C_{i} \bmod \left[T_{i-1}\right]$ time units rather than the first time unit as in [16]. This is due to the particular release of tasks.

4: $\quad$ For the $T_{i}$-mesoid $\mathcal{M}_{i}^{b, 2}$ resulting from the previous step, build the corresponding universe $X_{i}^{2}$ which consists of the ordered set of all availabilities of $\mathcal{M}_{i}^{b, 2}$. Notice that this set corresponds to the set of all possible values that the WCET $C_{i}$ of task $\tau_{i}$ can take in $\mathcal{M}_{i}^{b, 2}$.

5: Since $\tau_{i}$ is potentially schedulable, i.e. its WCET $C_{i} \in X_{i}^{2}$, we must verify that it is actually schedulable. Clearly, if $C_{i} \notin X_{i}^{2}$, then task $\tau_{i}$ is not schedulable because the deadline of the task is exceeded.

6: $\quad$ Determine the response time $R_{i}^{k}$ of task $\tau_{i}$ in its $k^{t h}$ instance, i.e. in the $k^{t h} T_{i}$-mesoid. This is obtained by summing $C_{i}$ with all the consumptions prior to $C_{i}$ in the corresponding mesoid. Deduce the worst-case response time $R_{i}$ of task $\tau_{i}$.

$$
R_{i}=R_{i}^{2}
$$

It is worth noticing that task $\tau_{i}$ is schedulable if and only if $\mathrm{R}_{i} \leq D_{i}$.
7: $\quad$ If $R_{i} \leq D_{i}$, then build $\mathcal{M}_{i}^{a, 2}=g\left(\mathcal{M}_{i}^{b, 2}\right)$, increment $i$, and go back to step 2 as long as there remain potentially schedulable tasks in the system.

8: If $R_{i}>D_{i}$, then the system $\left\{\tau_{i}=\left(C_{i}, T_{i}\right)\right\}_{1 \leq i \leq n}$ is not schedulable.

\section{9: end for}

Thanks to the above algorithm, a system of $n$ tasks $\left\{\tau_{i}=\left(C_{i}, T_{i}\right)\right\}_{1 \leq i \leq n}$, with harmonic periods and first released such that $r_{i}^{1}=r_{i-1}^{1}-C_{i}$, is schedulable if and only if

$$
R_{i}=R_{2}^{2} \leq D_{i} \quad \forall i \in\{1,2, \cdots, n\}
$$

\subsection{Computation of $\alpha$}

The value of $\alpha$ is given by: $\alpha=\max _{1 \leq i \leq n}\left(\frac{R_{i}}{T_{i}}\right)$ We recall that for the synchronous scenario, the worst case response time of task $\tau_{i}$ is given by:

$$
R_{i}=C_{i}+\sum_{j \in h p(i)}\left\lceil\frac{R_{i}}{T_{j}}\right\rceil C_{j}
$$

\section{Example}

Let us consider $\left\{\tau_{1}, \tau_{2}, \tau_{3}, \tau_{4}\right\}$ to be a system of four tasks with harmonic periods and first released such that $r_{i}^{1}=$ $r_{i-1}^{1}-C_{i}$. The characteristics are defined in table 1 .

\section{Table 1. Characteristics of the tasks}

\begin{tabular}{|c|c|c|}
\hline & $C_{i}$ & $T_{i}$ \\
\hline$\tau_{1}$ & 2 & 5 \\
\hline$\tau_{2}$ & 4 & 15 \\
\hline$\tau_{3}$ & 5 & 30 \\
\hline$\tau_{4}$ & 7 & 60 \\
\hline
\end{tabular}

The shorter the period of a task is, the higher its level is. Thus, as depicted in table $1, \tau_{1}$ has the highest level and task $\tau_{4}$ the lowest level. Thanks to our scheduling algorithm, for task $\tau_{1}$ whose first release time is $r_{1}^{1}=0$, we have

$$
\tau_{1}:\left\{\begin{array}{l}
\mathcal{M}_{1}^{b, 2}=\{1,2,3,4,5\} \\
R_{1}=2 \\
\mathcal{M}_{1}^{a, 2}=\{(2), 1,2,3\}
\end{array}\right.
$$

$\gamma_{2}=T_{2}-C_{2} \bmod \left[T_{1}\right]=15-4 \bmod [5]=1$, thus for task $\tau_{2}$ whose first release time is $r_{2}^{1}=r_{1}^{1}-C_{2}=-4$, we have

$$
\tau_{2}:\left\{\begin{array}{l}
\mathcal{M}_{2}^{b, 2}=\{(1), 1,2,3,(2), 4,5,6,(2), 7,8,9,(1)\} \\
R_{2}=4+2+1=7 \\
\mathcal{M}_{2}^{a, 2}=\{(7), 1,2,(2), 3,4,5,(1)\}
\end{array}\right.
$$

$\gamma_{3}=T_{3}-C_{3} \bmod \left[T_{2}\right]=30-5 \bmod [15]=10$, thus for task $\tau_{3}$ whose first release time is $r_{3}^{1}=r_{2}^{1}-C_{3}=-4-5=-9$, we have

$\tau_{3}:\left\{\begin{array}{l}\mathcal{M}_{3}^{b, 2}=\{(1), 1,2,3,(8), 4,5,(2), 6,7,8,(8), 9,10,(1)\} \\ R_{3}=5+8+1=14 \\ \mathcal{M}_{3}^{a, 2}=\{(16), 1,2,3,(8), 4,5,(1)\}\end{array}\right.$ 
$\gamma_{4}=T_{4}-C_{4} \bmod \left[T_{3}\right]=60-7 \bmod [30]=23$, thus for task $\tau_{4}$ whose first release time is $r_{4}^{1}=r_{3}^{1}-C_{4}=-9-7=-16$, we have

$\tau_{4}:\left\{\begin{array}{l}\mathcal{M}_{4}^{b, 2}=\{(4), 1,2,(17), 3,4,5,(8), 6,7,(17), 8,9,10,(4)\} \\ R_{4}=7+8+17+4=36 \\ \mathcal{M}_{4}^{a, 2}=\{(53), 1,2,3,(4)\}\end{array}\right.$

Consequently, the set of tasks $\left\{\tau_{1}, \tau_{2}, \tau_{3}, \tau_{4}\right\}$ with harmonic periods and first released such that $r_{i}^{1}=r_{i-1}^{1}-C_{i}$ is schedulable. The schedule with the above characteristics is depicted in figure 2 ,

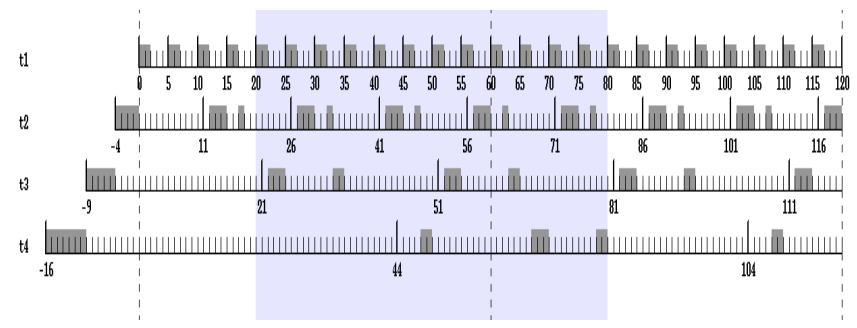

Figure 2. Execution of a set of harmonic tasks with $r_{i}^{1}=$ $r_{i-1}^{1}-C_{i}, \quad \forall i \in\{2, \cdots, 4\}$

whereas the schedule of the same set of tasks released simultaneously is depicted in figure 4.

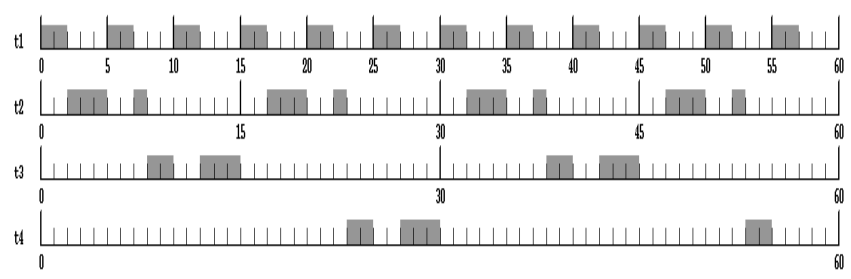

Figure 3. Execution of a set of harmonic tasks with $r_{i}^{1}=$ $0 \forall i \in\{1, \cdots, 4\}$

It is worth noticing here the large variation between the two scenarios in terms of the tasks' response times. As a matter of fact, the worst case response time of task $\tau_{4}$ in figure 2 is 36 whereas it is 55 in figure 4. This phenomenon is even more apparent in the next section with the experimental results where we gradually and uniformly decrease the value of the relative deadlines for all tasks by the same factor to highlight the advantage of our approach.

\begin{tabular}{|c|c|c|}
\hline Tasks & $R_{i}^{\text {synchronous }}$ & $R_{i}^{\text {asynchronous }}$ \\
\hline$\tau_{1}$ & 2 & 2 \\
\hline$\tau_{2}$ & 8 & 7 \\
\hline$\tau_{3}$ & 15 & 14 \\
\hline$\tau_{4}$ & 55 & 36 \\
\hline
\end{tabular}

This leads us to obtain $\alpha^{\text {synchrnous }}=$ $\max (2 / 5,8 / 15,15 / 30,55 / 60)=0.91$ whereas $\alpha^{\text {asynchrnous }}=\max (2 / 5,7 / 15,14 / 30,36 / 60)=0.60$, which means the improvement performed in this case is of $34.54 \%$

\section{Experimental results}

In this section we present some experimental results comparing the minimum deadline reduction factor $\alpha$ obtained in the synchronous scenario and in our particular asynchronous scenario. We consider harmonic tasks scheduled with the Deadline Monotonic algorithm.

We perform 10000 experiments for each graph, and every task set consists of $n=10$ harmonic tasks. The total utilization factor of the processor is randomly chosen between 0.7 and 1 for each task set. Hence, we can evaluate the gain of our specific asynchronous scenario defined in corollary 1 in section 2 , compared to the synchronous one. We set $\alpha=\frac{D_{i}}{T_{i}}$, and we gradually and uniformly decrease the value of the relative deadlines $D_{i}$ by the same factor for all tasks in each set. In both the synchronous and the asynchronous scenario, we plot the curves corresponding to the smallest value of $\alpha$, as a function of the total utilization factor of the processor, for the task set to remain schedulable. If the value of $\alpha$ is denoted $\alpha^{\text {synchronous }}$ in the synchronous scenario and $\alpha^{\text {asynchronous }}$ in our asynchronous scenario, the gain can be computed as follows:

$$
\text { gain }=\frac{\alpha^{\text {synchronous }}-\alpha^{\text {asynchronous }}}{\alpha^{\text {synchronous }}} \times 100
$$

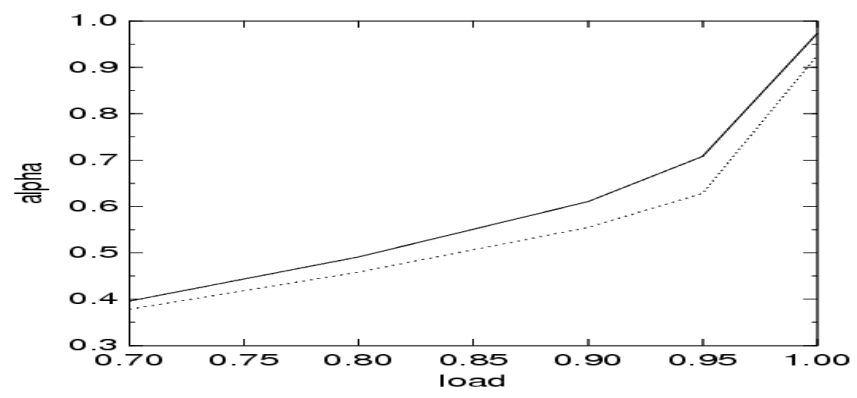

Figure 4. Value of $\alpha$ with our asynchronous scenario and with the synchronous scenario

In figure 4 , the curve in plain style represents the result obtained for $\alpha$ in our asynchronous case whereas the curve in dot style represents the result obtained in the synchronous case. In both cases, we start with a schedulable task set $\forall \tau_{i}, D_{i}=T_{i}$. From [15], $U \leq 1$ is a necessary and sufficient feasibility condition for the schedulability of an harmonic task set as tasks are scheduled with DM equivalent to RM when $\forall \tau_{i}, D_{i}=T_{i}$.

We can see that for a small load, we obtain almost the same $\alpha$ both in the synchronous and in the asynchronous 
cases. This is due to the fact that at small load the worst case response times of the tasks are less influenced by the release times of other tasks. As the load increases, the gain increases and reaches a maximum of $14,3 \%$ for $U=0.95$. Over the load $U=0.95$, the gain steadily decreases when $U$ tends to 1 and $\alpha$ tends to 1 . At high loads, the worst case response time of a task tends to its period and thus $\alpha$ tends to 1 . In this latter case, the improvement obtained with our asynchronous scenario becomes less significant.

\section{Conclusion}

In this paper, we have studied the sensitivity of the deadlines for periodic tasks when the tasks are scheduled with the Deadline Monotonic scheduling. We have considered a specific asynchronous task set and harmonic tasks. The asynchronous scenario we consider makes it possible to significantly reduce the complexity of the worst case response time computation. We have then considered the Mesoid approach to compute the worst case response time of a task in an asynchronous scenario. We have used the Mesoid approach to compute the minimum deadline reduction factor. We have proved by extensive simulations that the gain in terms of deadline reduction can reach $14,3 \%$ with our particular asynchronous scenario compared to the synchronous scenario. This makes it possible to better control the jitter of the tasks when considering control loops.

\section{References}

[1] S. Baruah, R. Howell, and L. Rosier. Algorithms and complexity concerning the preemptive scheduling of periodic real-time tasks on one processor. Real-Time Systems, Vol. 2, pp. 301-324, 1990.

[2] K. Tindell, A. Burns, and A. J. Wellings. Analysis of hard real-time communications. Real-Time Systems, Vol. 9, pp. 147-171, 1995.

[3] L. George, N. Rivierre, and M. Spuri. Preemptive and non-preemptive scheduling real-time uniprocessor scheduling. INRIA Research Report, No. 2966, September 1996.

[4] Giorgio Buttazzo Enrico Bini, Marco Di Natale. Sensitivity Analysis for Fixed-Priority Real-Time Systems. Proceedings of the 18th Euromicro Conference on Real-Time Systems (ECRTS'06), Dresden, Germany July 5-7, 2006.

[5] Ismael Ripoll Patricia Balbastre and Alfons Crespo. Optimal deadline assignment for periodic real-time tasks in dynamic priority systems. Proceedings of the 18th Euromicro Conference on Real-Time Systems (ECRTS'06), Dresden, Germany July 5-7, 2006.

[6] E. Bini and G. Buttazzo. The Space of EDF Feasible Deadlines. Proceedings of the 19th Euromicro Conference on Real-Time Systems (ECRTS'07), Pisa, Italy July 4-6 2007.

[7] M. Joseph and P. Pandya. Finding response times in a realtime system. BCS Comp. Jour., 29(5), pp. 390-395,, 1986.
[8] M. Grenier, J. Goossens, and N. Navet. Near-optimal fixed priority preemptive scheduling of offset free systems. Proc. of the 14th International Conference on Network and Systems (RTNS'2006), Poitiers, France, May 30-31, 20062006.

[9] Giorgio Buttazzo Enrico Bini. Schedulability Analysis of Periodic Fixed Priority Systems. IEEE Transactions On Computers, Vol. 53, No. 11, Nov.2004.

[10] J.P. Lehoczky. Fixed priority scheduling of periodic task sets with arbitrary deadlines. Proceedings 11th IEEE Real-Time Systems Symposium, pp 201-209, Dec. Lake Buena Vista, FL, USA, 1990.

[11] J. Y. T. Leung and M.L. Merril. A note on premptive scheduling of periodic, Real Time Tasks. Information Processing Letters, Vol 11, num 3, Nov. 19980.

[12] Annie Choquet-Geniet and Emmanuel Grolleau. Minimal schedulability interval for real-time systems of periodic tasks with offsets. Theor. Comput. Sci., 310(1-3):117-134, 2004.

[13] N. C. Audsley. Optimal priority assignment and feasibility of static priority tasks with arbitrary start times. Dept. Comp. Science Report YCS 164, University of York, 1991.

[14] J. Goossens. Scheduling of offset free systems. Real-Time Systems, 24(2):239-258, March 2003.

[15] G. C. Buttazzo. Rate Monotonic vs. EDF: Judgment Day. Real-Time Systems, 29, 5-26, 2005.

[16] P. Meumeu and Y. Sorel. Extending rate monotonic analysis with exact cost of preemptions for hard real-time systems. In Proceedings of 19th Euromicro Conference on Real-Time Systems, ECRTS'07, Pisa, Italy, July 2007.

[17] S. Baruah, A. K. Mok, and L. Rosier. Preemptively scheduling hard real-time sporadic tasks on one processor. Proceedings of the 11th Real-Time Systems Symposium, pp. 182-190, 1990.

[18] L. C. Liu and W. Layland. Scheduling algorithms for multiprogramming in a hard real time environment. Journal of $A C M$, Vol. 20, No 1, pp. 46-61, January 1973.

[19] J. Goossens. Scheduling of Hard Real-Time Periodic Systems with Various Kinds of Deadline and Offset Constraints. $\mathrm{PhD}$ thesis, Université Libre de Bruxelles, 1998.

[20] C.L. Liu and J.W. Layland. Scheduling algorithms for multiprogramming in a hard-real-time environment. Journal of the ACM, 1973.

[21] A.K. Mok S.K. Baruah and L.E. Rosier. Preemptively scheduling hard realtime sporadic tasks on one processor. In proc. 11th IEEE Real-Time Systems Symposium, 1990.

[22] Joseph Y.-T. Leung and M. L. Merrill. A note on preemptive scheduling of periodic, real-time tasks. Information Processing Letters, 1980.

[23] J. Leung and Whitehead J. On the complexity of fixedpriority scheduling of periodic real-time tasks. Performance Evaluation(4), 1982. 